\title{
Identification of the abuse of Performance-enhancing Agents in Medico-legal Investigations; Based on a Death of a Body-builder
}

\author{
KM Tikiri Bandara Gunathilake ${ }^{1 *}$, Muditha Vidanapathirana ${ }^{2}$ \\ ${ }^{1}$ Office of the Judicial Medical Officer, Base Hospital, Awissawella, Sri Lanka, \\ ${ }^{2}$ Depratment of Forensic Medicine, Faculty of Medical Sciences, University of Sri Jayewardenepura, Sri Lanka
}

\begin{abstract}
Introduction
Sportsmen abuse Performance-enhancing agents (PEA) to enhance their muscle mass and strength, while the side effects PEA may cause consequences and sudden deaths. However, the relatives usually are not aware of PEA-abuse, because most PEAs are purchased by the sportsmen from black-market. Therefore, the pathologists might miss PEAabuse and may dispose of the postmortem with a wrong cause of death. This concept introduces a guideline to identify PEA-abuse and to give recommendations for the proper medico-legal investigation of deaths due to PEA-abuse. A 37year-old bodybuilder who had a healthy-lifestyle died suddenly. Though the relatives were unaware, the Forensic pathologist profiled the findings and suspected PEA-abuse. The scene was visited but did not find PEA. Family physician revealed the abuse of PEA such as testosterone, cutting steroids, insulin-like growth factor and caffeine, and has had elevated liver enzymes. A high-risk autopsy was conducted. The face had acne and heart had concentric hypertrophy. Histopathology showed hypertrophy of the cardiac muscle and lung parenchyma and fatty liver. Therefore, we propose a postmortem guideline to identify PEA-abuse among sportsmen; (1) Establish seven PEAabuse profiles; Social, physical, psychological, clinical, sports, pathological and toxicological, (2) Before autopsy, to identify the types of PEA (i) Contact the physicians and (ii) visit the scene, (3) At the autopsy, use special approaches; (i) Consider as high-risk autopsy and (ii) If gene doping is suspected, do molecular autopsy. (4) To ascertain COD, consider abuse profiles, scene and autopsy findings. (5) For prevention, organize awareness programmes and (6) to prevent premature deaths recommend to employ a clinical screening tool.
\end{abstract}

Keywords: Autopsy, hypertrophy, guideline, PEA abuse, profile, sportsmen

Received: 27 May 2019, Revised version accepted: 20 June 2019, Published: 30 June 2018. *Corresponding author: Gunathilake KMTB, $\triangle$ Email: Tikiriii_bandara@yahoo.com, (D) https://orcid.org/0000-0002-8485-4550

Cite this article as: Gunathilake KMTB, Vidanapathirana M. Identification of the abuse of Performance-enhancing Agents in Medico-legal Investigations; Based on a Death of a Body-builder. Medico-Legal Journal of Sri Lanka, 2019;7(1): 15-21.

DOI: http://doi.org/10.4038/mljsl.v7i1.7389

Copyright: @ 2019 with the Medico-legal Journal of Sri Lanka.

This is an open-access article distributed under the terms of the Creative Commons Attribution 4.0 International License, which permits unrestricted use, distribution and reproduction in any medium provided the original author and source are credited.

\section{Introduction}

Competitive or recreational sportsmen and bodybuilders tend to abuse Performance-enhancing agents (PEA) to enhance muscle mass, muscle shaping or performance. There are many examples of deaths of body-builders due to the abuse of PEA in sports literature. In 2017, steroid-related deaths of strong body-builders such as Mike Matarazzo, Dan Puckett, Scott Klein, Robert Benevento, Trevor Smith and many more were reported. ${ }^{[1]}$ PEA is defined as any substance or drug that, when taken, gives a sportsman an unfair advantage relative to a "clean" sportsman. ${ }^{[2]}$ Lance Armstrong, once popular as a cycling superman, later became the greatest cycling fraud when he acknowledged that he was doping at all the international competitions. The other famous sports figures who lost their carriers due to PEA abuse were Marian Jones and Ben Johnson.

Human beings have anatomically and physiologically balanced systems. With the development of science, many drugs and chemicals have been synthesized artificially which could change this delicate balance of the body. The decade of 1930 was a milestone in endocrinology, because of the discovery of testosterone as the predominant androgen in humans and used as the hormone replacement therapy of androgen deficient men. ${ }^{[3]}$ In the next ten years, it was established that the testosterone administration is associated with gains of skeletal muscle mass and strength. ${ }^{[4]}$ Now it is a growing problem among sports 
communities and the PEAs are far more prevalent than anyone is willing to admit. ${ }^{[5]}$

The objectives of the abuse of PEA by the athletes and body-builders include (1) to increase growth (by using anabolic drugs such as Anabolic-androgenic steroids (AAS), recombinant human Growth hormone $(\mathrm{GH})$ and Insulin growth factor-1 (IGF-1), (2) to increase activity (by using stimulants such as caffeine, amphetamine, ephedrine, thyroxin etc.), (3) to increase endurance (by using endurance enhancement agents), (4) to increase memory (by using nootropics), (5) to increase blood (by using blood boosters, blood doping or erythropoietin), (6) to reduce stress (by using adaptogens), (7) to reduce pain (by using painkillers), (8) to reduce anxiety and fear (by using sedatives and anxiolytics) and (9) to reduce weight (by using laxatives or diuretics). ${ }^{[6]}$

Almost all PEAs give temporally benefits but the side effects cause serious problems. For example, GH and AAS will not only cause hypertrophy of the skeletal muscles but also cause changes in all the body systems, including cardiovascular system (premature atherosclerosis, cardiac muscle hypertrophy, changes in endothelium etc.), respiratory system (alveolar hypertrophy), renal, reproductive and psychological changes and could lead to sudden death at any time. ${ }^{[7]}$

The misuse of PEA produces well-built, very active and energetic people. However, almost all of their body systems are maintained artificially and the ill effects of PEA could cause sudden deaths at any time. In this context, the forensic pathologist must possess a better knowledge to suspect and identify the abuse of PEAs, collect relevant facts and to interpret them correctly. For example, if a forensic pathologist finds a young person with a hypertrophied heart, one of the main causes of deaths could be hypertrophic obstructive cardiomyopathy (HOCM). Unless the deceased had previously revealed the PEA misuse, the relatives or guardians may not know about it, because most of the PEAs are purchased illegally or from the black market. ${ }^{[8]}$ This will be a common problem in the future, as there is an increasing number of bodybuilding practices among the young generation. Although the use of PEA in sports has been banned, it is very difficult to control recreational use.

If dies suddenly, they are subjected to autopsies, however, in the absence of prior knowledge regarding misuse of PEA, the forensic pathologist might miss the possibility of PEA abuse especially in recreational sports, and the body may be disposed of with a common and wrong cause of death such as HOCM.

\section{Objectives}

Therefore, the following case discussion was performed based on sudden death of a young bodybuilder to formulate a method to identify the abuse of PEAs in sudden deaths of sportsmen and to give recommendations for the proper medico-legal investigation of deaths due to misuse of PEAs.

\section{Background history}

A 37-year-old young, male, body-builder died suddenly and unexpectedly in his home. He was a father of two children and an owner of three bodybuilding sports centres.

According to the wife, the deceased's social history showed that he was crazy over exercises and performed weight lifting vigorously, and spent a long time in gyms. Further, he had taken a large number of vitamins and food supplements, however, the wife or the relatives did not know about taking any parenteral or oral Performance-enhancing agents (PEA). He was a non-alcoholic and a non-smoker and had an apparently "healthy lifestyle". He had been a strict vegetarian and had heavy meals. He had not been on drugs for any significant illness. The past medical history was unremarkable and there was no family history of sudden deaths.

The wife expressed that the deceased was a highly active person. However, it was revealed that he had a rapid gain in muscle and weight.

Therefore, having identified the possible misuse of PEA, the forensic pathologist used a novel approach and used extra protective measures in the medico-legal investigation.

A scene visit was performed before the autopsy and recovered some dietary supplements, however, there were no PEAs such as Anabolic androgen steroids (AAS), Insulin-like Growth factor-1 (IGF-1) or Growth hormone $(\mathrm{GH})$ at the scene.

Afterwards, the pathologist contacted his personal physician who revealed that the deceased was using few cycles of testosterone and IGF-1 injections, several cutting steroids, elements containing a high dose of caffeine (275mg per serve), multiple protein supplements and pre-workout booster dietary supplements. When the reports of the past routine medical reports were perused, nothing abnormal was found other than the moderate elevation of SGOT and SGPT.

In this case, the autopsy was considered a high-risk autopsy and strict preventive measures were taken. 
The body was of a well-nourished, well-built male with a height of 6 feet and 4 inches and $\mathrm{h} 16$ weight was $134 \mathrm{~kg}$. Externally, he had acne on unc raue (Fig. 01), well-built muscles and few tattoos. There were no external or internal injuries.

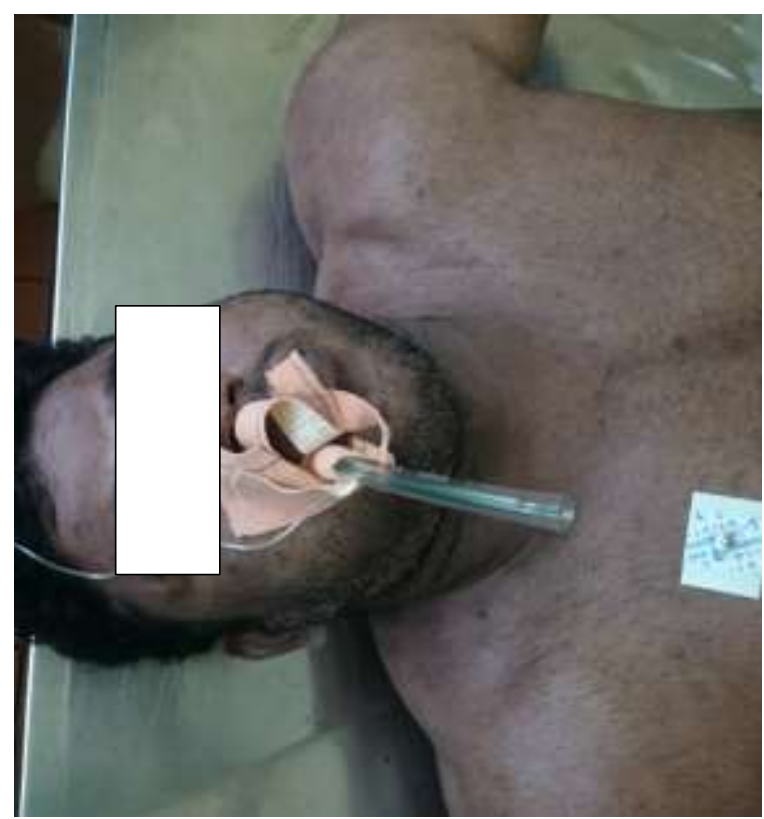

Figure 01: Well-built and muscular body. Note the acne on the face.

All organs were dissected and the heart muscle was hypertrophied and both the ventricles were disproportionately enlarged. (Fig. 02) The atria appeared enlarged and the ventricles represented $3 / 4^{\text {th }}$ of the size of the heart. The heart had concentric hypertrophy of all four chambers and the thickness of the left ventricle was $2 \mathrm{~cm}$. The left ventricular trabeculae appeared prominent and there was no evidence of valvular heart disease. The coronaries and aorta had few atheromatous plaques but did not cause a significant occlusion. The weight of the heart was $550 \mathrm{~g}$.

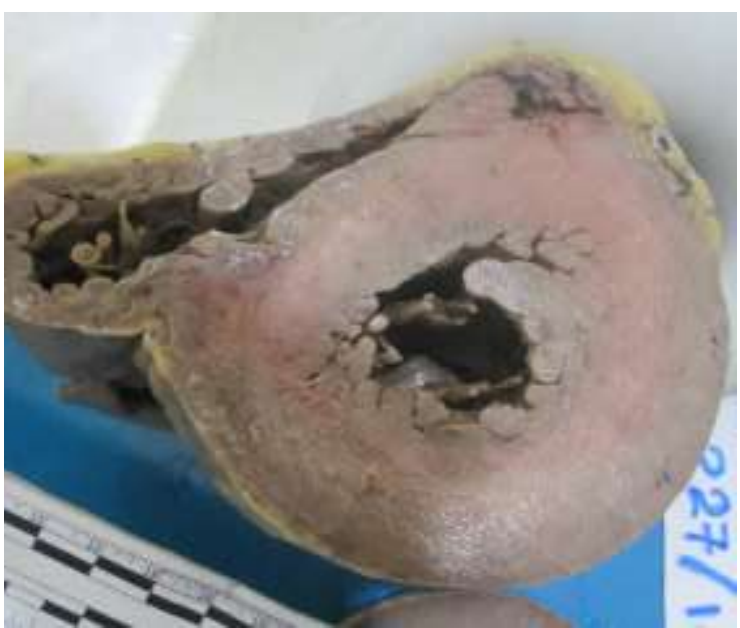

Figure 02: Hypertrophied ventricles

Blood and tissues were sent for routing toxicological analysis and for PEAs such as testosterone (AAS) and IGF-1. Alcohol was negative and the results of PEAs were awaiting.

The histopathological studies of all the organs were performed and had hypertrophy of the cardiac muscle fibres, fibrosis and hypertrophy of the lung parenchyma and fatty changes of the liver. The cause of death was given as hypertrophy of the heart probably due to long term use of PEA.

\section{Discussion}

Performance-enhancing agents (PEA) abuse in sports is a growing problem, especially among the young population. In the recent past in Sri Lanka, there is an increasing number of bodybuilding centers and gymnasiums in every corner of the country targeting the young generation. Other than the exercise schedules, they tend to misuse Performanceenhancing agents (PEA) to gain maximum benefits within a short period of time. Almost all of these drugs have harmful effects on the body; physically, psychologically and socially. Some of those side effects could even lead to death. For example, due to physical effects of PEA such as cardiac hypertrophy, fibrosis, myocardial infarctions, arrhythmias, coronary heart disease and due to psychological effects such as increased suicide tendency can kill.. ${ }^{[9]}$ The competitive body-builders may also use potent diuretics like frusemide to induce diuresis to prevent drug misuse being identified. However, diuresis can lead to electrolyte imbalance and can cause death due to arrhythmia or fits etc. ${ }^{[10]}$

The next medico-legal issue is the ascertainment of the cause of death (COD). Therefore, this discussion is conducted on the following issues; (1) Profiling of 
PEA abuse; (1.1) Social profile, (1.2) physical profile, (1.3) psychological profile, (1.4) clinical profile, (1.5) sports profile, (1.6) pathological profile and (1.7) toxicological profiling of PEA abuse, ${ }^{[11]}$

(2) General forensic pathological issues of PEA abuse and (3) Preventive measures in PEA abuse.

In this case, since the deceased was a body-builder who died suddenly, the pathologist needed to look for the contribution of PEA in such death. The wife and the relatives of the deceased did not know about the PEA abuse. In such instances, a special evaluation should be done, based on the above seven profiles, to identify the PEA abuse and the COD.

\section{(1.1) Social profile relevant to PEA abuse}

The pathologist has to see whether the deceased has a social profile relevant to PEA abuse. For example, whether the deceased has (1) obsessions with exercise, (2) does vigorous weight lifting, (3) spends long time in gymnasiums or health clubs, (4) recent changes in friends, (5) takes large amounts of vitamins and food supplements, (6) takes very high-calorie intake, (7) whether leads a "healthy lifestyle" and (8) whether on any other medicinal drugs. In this case, the deceased showed several such social profiles of PEA abuse. He engaged in vigorous weight lifting, spent a long time in gyms and had large amounts of vitamins and food supplements, In addition, he was a teetotaler with a 'healthy lifestyle', ate large healthy diets and was not on any other medicinal drugs. Those features favoured PEA abuse.

\section{(1.2) Physical profile relevant to PEA abuse}

The pathologist has to see whether the deceased has a physical profile relevant to PEA abuse. For example, (1) rapid weight gain with muscle development, (2) increase or decrease of body hair, and (3) deepening of the voice. In this case, the deceased had a wellnourished, well-built body with well-built muscles which favoured the physical profile of PEA abuse.

\section{(1.3) Psychological profile relevant to PEA abuse}

The pathologist has to see whether the deceased has a psychological profile of PEA abuse. For example, (1) increased aggression, (2) hyperactivity, (3) auditory hallucinations, (4) depression, (5) anxiety and (6) suicidal thoughts. In this case, the psychological profile revealed that he was a hyperactive person.

\section{(1.4) Clinical profile relevant to PEA abuse}

The pathologist has to see whether the deceased has a clinical profile relevant to PEA abuse. For example, (1) acne, (2) elevation of blood pressure, (3) complaining of stomach aches, (4) jaundice and (5) oedema of the extremities and (6) significant antemortem clinical test reports. In this case, he was apparently well and was not on any medicinal drugs. Fig. 01 shows that he had acne on his face. Antemortem reports revealed elevation of SGOT and SGPT. Therefore, the clinical profile favoured the abuse of PEA such as AAS.

\section{(1.5) Sports profile relevant to PEA abuse}

When a forensic pathologist involves with an autopsy of a competitive or recreational sportsman, he/she should reconstruct the type of PEA abuse by considering the type of the sport/s the deceased engaged in. For example, (1) Anabolic androgenic steroids (AAS) including testosterone are used in bodybuilding, wrestling, basketball etc. Cutting steroids are used for cutting effects of the muscles and are used by body-builders. ${ }^{[12]}$ (2) Caffeine, amphetamine, cocaine etc. are used to stimulate and increase CNS alertness and are used by body-builders etc. (3) GH and IGF-1 are used in bodybuilding and the (4) laxatives and diuretics to reduce the body weight in body-builders and boxing. ${ }^{[10]}$ In this case, the deceased had been performing recreational bodybuilding and the possible abused PEA could be anabolic drugs, CNS stimulants, protein and dietary supplements and quick energy boosters such as insulin growth factors (IGF-1). Therefore, the deceased's use of testosterone, cutting steroids elements, insulin-like growth factor-1 (IGF-1), high dose of caffeine, multiple protein supplements and pre-workout booster dietary supplements are compatible with his recreational bodybuilding.

\section{(1.6) Pathological profile relevant to PEA abuse}

During the autopsy, the pathologist has to see whether the deceased has a pathological profile relevant to PEA abuse. For example, (1) cardiac hypertrophy (2) lung parenchyma hypertrophy, (3) skeletal muscle hypertrophy, (4) atherosclerosis, (5) liver fatty changes and peliosis hepatis.

When cardiac hypertrophy is present, it is relatively difficult to differentiate from the $\mathrm{GH}$ and AAS abuse. Therefore, history such as premature deaths in the family and their autopsy reports are important. There is asymmetric hypertrophy in HOCM, with septal hypertrophy of $15 \mathrm{~mm}$ thickness, but $2 \%$ of trained athletes have a similar pattern. There is a distinctive histopathological pattern of the disarray of myocardial cells in HOCM. However, the differentiation between pathological and physiological hypertrophy is difficult for trained athletes. The upper limit to which the thickness of the left ventricle may be increased by training appears to be $16 \mathrm{~mm}$. Therefore, athletes with a wall thickness of more than $16 \mathrm{~mm}$ are likely to have 
primary forms of pathological hypertrophy, such as hypertrophic cardiomyopathy, possibly associated with long-term AAS abuse. ${ }^{[13,14]}$ Other than this, in general, considering the whole picture such as the habits, body stature and build, background and most importantly abnormalities in other body systems such as in heart and liver are seen.

In the vascular system, the premature formation of atheromatous plaques could be seen. Considering all, it may be possible to come to the conclusion of cardiac pathology as the targeted main pathology which leads to death due to AAS.

In this case, the ventricular hypertrophy was $2 \mathrm{~cm}$ and histopathology of heart did not show fibre disarray. Those features favoured AAS abuse. The family physician confirmed that he was on testosterone, cutting steroids and are compatible with AAS abuse.

\section{(1.7) Toxicological profiling of PEA abuse}

The forensic pathologist has to send samples for common poisons such as alcohol and for alleged types of PEA abuse.

However, the interpretation of toxicological findings in post-mortem examination is difficult as most of the PEAs are normal components of the body such as testosterone (AAS), Insulin (IGF-1), Growth hormone $(\mathrm{GH})$, erythropoietin, or drugs which may be used in normal clinical practice such as salbutamol. In this case, the blood alcohol was negative and other PEA results were awaiting.

In some instances, the PEA test reports may come negative. This could be due to non-availability of sophisticated PEA analysis techniques in autopsy practice in Sri Lanka, however, they are used in the detection of sports doping in developed countries. The other reason is the use of such drugs in unexpected patterns such as "Drug holidays". Sometimes, the drug is stopped long before the onset of the competition or tailed off towards the competition. Some use PEA in cycles and in between the cycles, the drug may not be detected in the blood.

Therefore, reviewing the antemortem laboratory finding of PEA tests or clinical reports such as lipid profile with decreased HDL and TSH, increased LDL, increased LH, TG, Glucose, haemoglobin ( $\mathrm{Hb}$ ) etc. will give clues regarding the possible PEA abuse. For example, in the administration of erythropoietin, there will be elevated levels of Haemoglobin in antemortem reports. Therefore, the consideration of the type of anti-mortem reports and the possible expected outcome could give valuable clues regarding the possible PEA abuse. However, in this case, only the liver enzymes such as SGOT and SGPT had been elevated and favoured AAS or other PEA abuse.

\section{(2) General forensic pathological issues of PEA abuse}

The PEA abuse can cause special issues at the crime scene and the autopsy investigations. Since these drugs are restricted or banned and are purchased from the black-market can lead to medico-legal issues such as not being able to recover at the crime scene investigation and non-availability of a reliable history of PEA abuse during the autopsy investigation. Similarly, in this case, at the autopsy investigation, there was no such history of PEA abuse. Further, the scene investigation did not reveal any PEAs. Therefore, the forensic pathologist had to reach the family physician to acquire the relevant past history of the usage of parenteral and oral PEAs.

Another medico-legal issue is the possibility of the transmission of deadly diseases during the autopsy investigation of such deaths. For example, the GH had been produced by human pituitary extracts in the past, however, it is now produced by using other animal tissue extracts. Therefore, it has a risk of transmitting diseases such as Creutzfeldt-Jakob disease. ${ }^{[7]}$ Moreover, if the needles are shared by the athletes or body-builders, there is an additional risk of HIV or $\mathrm{HBV}^{[10]}$. Since the pre-autopsy screening of such deadly diseases is not available in Sri Lanka, the forensic pathologist considered this autopsy investigation of the body-builder as a high-risk autopsy and took all the preventive measures.

Now there are new methods of acquiring PEA such as gene doping. Other than obtaining those drugs directly, there may be possibilities with advanced technology by inserting hormone-producing genes. ${ }^{[15]}$ Therefore, such autopsies should be a molecular autopsy and samples should be taken for genetic studies to investigate gene doping. However, in this case, we did not take samples for gene doping because there had been no history of employment of such methods by the deceased.

Another newer method of introducing PEAs is using a viral vector such as adenovirus and produce druginduced tumours that produce hormones such as GH. Therefore, a meticulous histopathological investigation of all organs should be done to investigate the drug-induced tumours producing hormones. In this case, all organs were dissected and histopathology samples were taken from all the organs and investigated. 


\section{(3) Preventive measures in PEA abuse}

Because of the illegal nature of drug usage, young athletes or people will not attend to obtain treatments or investigations. Further, there are no education programs to highlight the side effects or fatal outcomes of abuse of PEA. Therefore, early recognition of cardiac complications by echocardiography, liver function tests etc. should be used for early identification and to take preventive measures. Screening programs exist in several countries such as USA, Italy, mainly to screen athletes to prevent sports-related deaths. Therefore, the introduction of such a PEA screening method for competitive and recreational sportsmen will be useful to prevent PEA abuse and to prevent premature deaths. ${ }^{[16]}$

\section{Conclusion}

Performance-enhancing agents (PEA) abuse among sports and recreation activities is a growing problem. Most of those PEA are banned or controlled in public use. Illegal nature of public usage may lead to problems in postmortem investigations such as nonavailability of evidence of PEA abuse in the preautopsy history and at the scene investigation.

Therefore, to identify the PEA abuse in the death investigation of sportsmen, we propose a new guideline.

(1) During the postmortem investigation, to identify PEA abuse, establish following seven (07) profiles; Social profile, physical profile, psychological profile, clinical profile, sports profile, pathological profile and toxicological profiles.

(2) Before the autopsy, to identify the types of PEA (a) Contact the physicians involved such as a family physician to identify the types of PEA abuse. (b) Perform a crime scene investigation preferably before the autopsy and look for evidence of PEA abuse.

(3) At autopsy, use special approaches; (a) consider the autopsy investigation as a high-risk autopsy and take preventive measures. (b) If the suspect was gene doping, do a molecular autopsy.

(4) To ascertain the COD, consider all seven profiles, scene and autopsy findings together.

(5) For prevention of PEA abuse, organize awareness programmes for the clinicians regarding the early diagnosis and for the sportsmen regarding the consequences of PEA abuse.
Finally, (6) to identify PEA abuse early and prevent premature deaths we also recommend employing an accepted clinical screening tool among sportsmen.

\section{Disclosure statement}

Conflicts of interests: The authors declare that they have no conflicts of interests.

Funding: None

\section{References}

1. Chronic Hunter, body-builders who died of steroids; http//www.youtube.com/ watch?v= bDATXTjBSW

2. Dandoy C, Rani S. Gereige RS. PerformanceEnhancing Drugs. Pediatr Rev. 2012 Jun; 33(6): 265-72. Doi: 10.1542/pir.33-6-265

3. Wilson JD, Androgen abuse by athletes, Endocr Rev,1988;9(2):188-9. DOI:

10.1210/edrv-9-2-181

4. Bhasin S, Cunningham GR, Hayes FJ, Matsumoto AM, Snyder PJ, Swerdloff RS, Montori VM; Task Force, Endocrine Society. J Clin Endocrinol Metab. 2010 Jun;95(6):2536-59. Doi: 10.1210/jc. 2009-2354.

5. Yesalis CE1, Barsukiewicz CK, Kopstein AN, Bahrke MS. Trends in anabolic-androgenic steroid use among adolescents. Arch Pediatr Adolesc Med. 1997 Dec;151(12):1197-206. PMID: 9412594

6. Haerinejad MJ, Ostovar A, Farzaneh MR, Keshavarz M. The Prevalence and Characteristics of Performance-Enhancing Drug Use Among Bodybuilding Athletes in the South of Iran, Bushehr. Asian J Sports Med. 2016 Jul 16;7(3):e35018. PMID: 27826400

7. Holt RI, Sönksen PH. Growth hormone, IGF-I and insulin and their abuse in sport. $\mathrm{Br} \mathrm{J}$ Pharmacol. 2008 Jun;154(3):542-56. Doi: 10.1038/bjp.2008.99.

8. Steven B. Karch, Pathology of drug abuse; $4^{\text {th }}$ Ed, Boca Raton: CRC press; 2009. p-613.

9. Hunter M. Athletes risk their lives by routine use of performance-enhancing drugs, says BMA. 
BMJ. 2002 Apr 13; 324(7342): 870. PMCID: PMC1172116

10. Mason JK, Perdue BN, Pathology of trauma; London: Arnold; 2000. p. 285-6.

11. Narducci WA, Wagner JC, Hendrickson TP, Jeffrey TP. Anabolic steroids--a review of the clinical toxicology and diagnostic screening. J Toxicol Clin Toxicol. 1990;28(3):287-310. PMID: 2231829

12. Ghigo E, Lanfranco F, stasberger CJ. Hormone use and abuse by athletes. London: Sphinger;2011. p.609.

13. Dickerman RD, Schaller F, McConathy WJ. Left ventricular wall thickening does occur in elite power athletes with or without anabolic steroid Use. Cardiology. 1998 Oct;90(2):145-8. DOI: 10.1159/000006834.

14. Pelliccia A, Maron BJ, Spataro A, Proschan MA, Spirito P. The upper limit of physiologic cardiac hypertrophy in highly trained elite athletes. N Engl J Med. 1991 Jan 31;324(5):295-301. DOI: 10.1056/NEJM199101313240504

15. Segura J, Gutiérrez-Gallego R, Ventura R, Pascual JA, Bosch J, Such-Sanmartin G, et al. Growth hormone in sport: beyond Beijing 2008. Ther Drug Monit. 2009 Feb;31(1):3-13. Doi: 10.1097/FTD. 0b013e318194cc94.

16. Maron BJ, Thompson PD, Puffer JC, McGrew CA, Strong WB, Douglas PS. et al. Cardiovascular pre-participation screening of competitive athletes. A statement for health professionals from the Sudden Death Committee (clinical cardiology) and Congenital Cardiac Defects Committee (cardiovascular disease in the young), American Heart Association. Circulation.1996;94(4):850-6. PMID:8772711 